\title{
Caracterização clínica-epidemiológica de pacientes em terapia de hemodiálise: Uma revisão integrativa
}

\author{
Clinical-epidemiological characterization of patients undergoing hemodialysis therapy: An \\ integrative review \\ Caracterización clínico-epidemiológica de pacientes en hemodiálisis: Una revisión integradora
}

Recebido: 20/03/2021 | Revisado: 29/03/2021 | Aceito: 03/04/2021 | Publicado: 14/04/2021

Marta Valeria Soares Chaves

ORCID: https://orcid.org/0000-0002-3797-3696 Centro Universitário de Ciências e Tecnologia do Maranhão, Brasil E-mail: martavaleria706@gmail.com

Hálmisson D’Árley Santos Siqueira

ORCID: https://orcid.org/0000-0001-9831-5892

Centro Universitário de Ciências e Tecnologia do Maranhão, Brasil

E-mail: halmisson@yahoo.com.br

Wenderson Costa da Silva

ORCID: https://orcid.org/0000-0001-6031-9775

Universidade Estadual do Maranhão, Brasil

E-mail: wendersoncosta09@hotmail.com

Thalia Jeovana da Silva Pereira

ORCID: https://orcid.org/0000-0003-0323-5318

Centro Universitário de Ciências e Tecnologia do Maranhão, Brasil

E-mail:thaliajeovana96@gmail.com

Karen Laís Azevêdo Oliveira Sousa

ORCID: https://orcid.org/0000-0002-0308-417X

Centro Universitário de Ciências e Tecnologia do Maranhão, Brasil

E-mail: Karen.lais05@gmail.com

Laine da Costa Almeida Barboza

ORCID: https://orcid.org/0000-0002-9801-1546

Centro Universitário de Ciências e Tecnologia do Maranhão, Brasil

E-mail: laynnealmeidah@gmail.com

Leonardo Carneiro Vieira

ORCID: https://orcid.org/0000-0002-5192-9018

Centro Universitário de Ciências e Tecnologia do Maranhão, Brasil

E-mail: leonardo.viera06@hotmail.com

Zuinglio Viana Mourão

ORCIR: https://orcid.org/0000-0003-2953-0825

Centro Universitário de Ciências e Tecnologia do Maranhão, Brasil

E-mail: zuinglioanaiv2@gmail.com

Genivaldo Pereira de Almeida Júnior

ORCIR: https://orcid.org/0000-0002-8661-5836

Centro Universitário de Ciências e Tecnologia do Maranhão, Brasil

E-mail: almeidajuniorgm@gmail.com

Pablo Henrique Costa Rocha

ORCIR: https://orcid.org/0000-0001-7433-8141

Centro Universitário de Ciências e Tecnologia do Maranhão, Brasil E-mail: pabloblayd64@gmail.com

João Felipe Tinto Silva

ORCID: https://orcid.org/0000-0003-3662-6673

Centro Universitário de Ciências e Tecnologia do Maranhão, Brasil

E-mail: felipetinto99@gmail.com

Linccon Fricks Hernandes

ORCID: https://orcid.org/0000-0002-7642-3080

Santa Casa de Misericórdia de Vitória, Brasil

E-mail: fricksjr@hotmail.com

Deusilene dos Santos Anjos

ORCID: https://orcid.org/0000-0001-7612-987X

Centro Universitário Planalto do Distrito Federal, Brasil

E-mail: Deusilene.cx@gmail.com

Ludmylla Lima da Conceição

ORCID: https://orcid.org/0000-0001-8732-4304

Centro Universitário de Ciências e Tecnologia do Maranhão, Brasil

E-mail: ludlimamylla@outlook.com 


\begin{abstract}
Resumo
O presente estudo objetivou analisar, por meio da literatura, as evidências científicas que envolvem os aspectos clinico-epidemiológicos de pacientes submetidos a terapia de hemodiálise. Trata-se de uma revisão integrativa da literatura, onde formulou-se a seguinte questão: Quais as evidências científicas sobre a caracterização clínica epidemiológica de pacientes submetidos ao processo de hemodiálise? Montou-se uma estratégia PICo na qual, por meio de descritores e palavras-chave foram consultadas as bases de dados PubMed da National Library of Medicine; BVS (Biblioteca Virtual da Saúde), e CINAHL (Cumulative Index to Nursing and Allied Health Literature). Utilizaram-se os estudos disponíveis em sua totalidade, publicados entre os anos de 2015 a 2020, nos idiomas português, inglês e espanhol. Foram incluídos treze estudos nesta revisão. Os treze estudos apresentaram a caracterização clínica-epidemiológica desses pacientes, prevalecendo: sexo masculino, idade de 20-59 anos, baixa escolaridade, baixa renda, com doenças de base hipertensão arterial e diabetes mellitus, as queixas mais frequentes associadas a hemodiálise, foram alterações físicas, cefaleia, câimbras, náuseas, vômitos, alterações mentais e alterações nos hábitos de vida. Portanto, espera-se contribuir para o alcance de uma verificação contínua da epidemiologia desses pacientes renais crônicos, visando a detecção precoce dessa patologia, como também as devidas condutas terapêuticas para evitar o desenvolvimento da doença, buscando minimizar o sofrimento desses pacientes, com foco sempre no cuidado integral dos indivíduos e a redução de desfechos desfavoráveis.
\end{abstract}

Palavras-chave: Pacientes; Perfil de saúde; Diálise renal.

\begin{abstract}
The present study aimed to analyze, through the literature, the scientific evidence that involves the clinical and epidemiological aspects of patients undergoing hemodialysis therapy. This is an integrative literature review, which asked the following question: What is the scientific evidence on the epidemiological clinical characterization of patients undergoing the hemodialysis process? A PICo strategy was set up in which, through descriptors and keywords, the PubMed databases of the National Library of Medicine were consulted; VHL (Virtual Health Library), and CINAHL (Cumulative Index to Nursing and Allied Health Literature). The studies available in their entirety, published between the years 2015 to 2020, in Portuguese, English and Spanish were used. Thirteen studies were included in this review. The thirteen studies presented the clinical and epidemiological characterization of these patients, prevailing: male gender, age 20-59 years, low education, low income, with underlying diseases hypertension and diabetes mellitus, the most frequent complaints associated with hemodialysis, were changes headache, cramps, nausea, vomiting, mental changes and changes in lifestyle. Therefore, it is expected to contribute to the achievement of a continuous verification of the epidemiology of these chronic kidney patients, aiming at the early detection of this pathology, as well as the appropriate therapeutic approaches to prevent the development of the disease, seeking to minimize the suffering of these patients, always focusing in the integral care of individuals and the reduction of unfavorable outcomes.
\end{abstract}

Keywords: Patients; Health profile; Renal dialysis.

\title{
Resumen
}

El presente estudio tuvo como objetivo analizar, a través de la literatura, la evidencia científica que involucra los aspectos clínicos y epidemiológicos de los pacientes sometidos a terapia de hemodiálisis. Se trata de una revisión integradora de la literatura, que planteó la siguiente pregunta: ¿Cuál es la evidencia científica sobre la caracterización clínica epidemiológica de los pacientes sometidos al proceso de hemodiálisis? Se estableció una estrategia PICo en la que, a través de descriptores y palabras clave, se consultaron las bases de datos PubMed de la Biblioteca Nacional de Medicina; BVS (Biblioteca Virtual en Salud) y CINAHL (Índice Acumulativo de Literatura en Enfermería y Afines en Salud). Se utilizaron los estudios disponibles en su totalidad, publicados entre los años 2015 a 2020, en portugués, inglés y español. Se incluyeron trece estudios en esta revisión. Los trece estudios presentaron la caracterización clínica y epidemiológica de estos pacientes, predominando: sexo masculino, edad 20-59 años, baja escolaridad, bajos ingresos, con enfermedades de base hipertensión y diabetes mellitus, las quejas más frecuentes asociadas a la hemodiálisis, fueron cambios en la cefalea, calambres, náuseas, vómitos, cambios mentales y cambios en el estilo de vida. Por tanto, se espera contribuir a la consecución de una verificación continua de la epidemiología de estos pacientes renales crónicos, con el objetivo de la detección precoz de esta patología, así como los abordajes terapéuticos adecuados para prevenir el desarrollo de la enfermedad, buscando minimizar el sufrimiento de estos pacientes, siempre enfocándose en la atención integral de las personas y la reducción de desenlaces desfavorables.

Palabras clave: Pacientes; Perfil de salud; Diálisis renal.

\section{Introdução}

Os rins são os principais aparelhos excretores e mediadores de substâncias endógenas e exógenas. O seu maior objetivo é ajustar os líquidos corporais e causar o equilíbrio hidroeletrolítico e acidobásico, para que não aconteça uma alteração que venha a desenvolver uma Lesão Renal Aguda (LRA) ou Doença Renal Crônica (DRC) (Albuquerque, 2017). 
A DRC é conhecida como uma complicação tanto para a saúde pública quanto para os usuários, a mesma restringe-se em lesões renais conhecidas pela perda progressiva e irreversível do funcionamento dos rins. É uma doença de elevada morbidade e mortalidade, assim como é caracterizada como um problema complexo que exige diversas abordagens no seu tratamento, faz-se necessário um manuseio mais racional dos recursos econômicos direcionado para essa terapêutica. A estadia dos pacientes no sistema de saúde gera grande custo e essa condição é o principal responsável pelos gastos com tratamento de DRC (Formiga, 2015).

O registro desses pacientes se faz através dos conjuntos de informações e a partir deles é viável traçar um perfil sociodemográfico dos usuários que fazem uso das terapias, considerando o crescente número de pacientes com DRC registrados nos sistemas de informações brasileiros e que necessitam de diálise. Portanto, é possível medir o tempo de sobrevida desses pacientes e a evolução desse indicador ao longo dos anos (Formiga, 2015).

No Brasil, em 2018, o número total estimado de pacientes em diálise era de 133.464. As estimativas das taxas de prevalência e incidência de pacientes em tratamento dialítico por milhão da população (pmp) foram de 640 e 204, respectivamente, com aumentos médios anuais de 23,5 pmp e 6 pmp para prevalência e incidência, respectivamente. A mortalidade bruta anual foi de 19,5\%. Dos pacientes prevalentes, 92,3\% estavam em hemodiálise e 7,7\% em diálise peritoneal, com 29.545 (22,1\%) em lista de espera para o transplante (Neves, Sesso, Thomé, Lugon, \& Nasicmento, 2020).

A hemodiálise é a terapia renal substitutiva (TRS) mais utilizada pelos portadores de DRC, é um procedimento realizado através do qual uma máquina retira o sangue do paciente e transfere para máquina, em que é realizada a diálise. O procedimento libera o corpo dos resíduos prejudiciais à saúde como o excesso de líquidos, normalmente as sessões são feitas de 3 a 4 vezes por semanas, com durabilidade de quatro horas por sessão (Galvão, Silva, \& Santos, 2018).

O paciente renal crônico apresenta estado de dependência ao tratamento, que é complicado, ajustando-se a uma condição predominante que provoca múltiplas mudanças diárias tanto para o cliente quanto para seus familiares. A prevenção, o tratamento e a avaliação das circunstâncias e danos modificáveis, como a obesidade, diabetes mellitus (DM), hipertensão arterial sistêmica (HAS), dislipidemia, patologia cardiovascular e tabagismo, precisam atingir determinações de acordo com as regras e os princípios do Ministério da Saúde (Aoyama, Santos, Sousa, Oliveira, \& Lima, 2019).

Com base no exposto, traçou-se a seguinte problemática: Quais evidências científicas mostram os aspectos clínicosepidemiológicos e perfil sociodemográfico de pacientes em terapia de hemodiálise?

Devido à grande elevação da doença, faz-se necessário a realização de estudos que abordem alguns aspectos relacionados ao tratamento, às limitações enfrentadas, descobrir os possíveis comprometimentos decorrentes destas situações, bem como as adaptações necessárias em suas vidas para a concretização do tratamento e quanto à prevenção de doenças.

O presente trabalho se justifica pela necessidade de um estudo para traçar o perfil de pacientes com DRC, para podermos conhecer o público usuário do serviço e principalmente como se comporta a sobrevida dos pacientes submetidos a esse tratamento, além disso, é importante para o planejamento de assistência buscando e promovendo melhorias na qualidade de vida dos pacientes. Podendo de alguma forma ajudar na terapia desses pacientes, sabendo como traçar medidas mais apropriadas e realizar um bom acompanhamento.

Frente ao exposto, objetivo geral dessa pesquisa foi analisar, por meio da literatura, as evidências científicas que envolvem os aspectos clinico-epidemiológicos de pacientes submetidos a terapia de hemodiálise. E especificamente descrever o perfil sociodemográfico dos pacientes em tratamento de hemodiálise; identificar as queixas associadas à hemodiálise; e determinar as principais etiologias associadas ao desenvolvimento da DRC e suas comorbidades. 


\section{Metodologia}

O presente estudo trata-se de uma pesquisa bibliográfica do tipo revisão integrativa da literatura. Este procedimento foi escolhido por possibilitar a síntese e análise do conhecimento científico já produzido sobre o tema "CARACTERIZAÇÃO CLÍNICA-EPIDEMIOLÓGICA DE PACIENTES EM TERAPIA DE HEMODIÁLISE: uma revisão integrativa”. Esta revisão utilizou a metodologia proposta no estudo de Oliveira et al. (2016).

O tema, determinou a construção da estratégia PICo, que representa um acrônimo para Paciente ou Problema (P), Interesse (I), Contexto (Co), na qual foi utilizada para a geração da questão norteadora desta revisão integrativa da literatura: "Quais evidências científicas mostram os aspectos clínicos-epidemiológicos e perfil sociodemográfico de pacientes em terapia de hemodiálise?"

Para a localização dos estudos relevantes, que respondessem à pergunta de pesquisa, utilizou-se de descritores indexados e não indexados (palavras-chave) nos idiomas português, inglês e espanhol. Os descritores foram obtidos a partir do Medical Subject Headings (MESH), dos Descritores em Ciências da Saúde (DeCS) e dos títulos Cumulative Index to Nursing and Allied Health Literature (CINAHL), como mostra o Quadro 1.

Consultou-se por meio de descritores e palavras-chave as bases de dados PubMed da National Library of Medicine; Biblioteca Virtual da Saúde (BVS), coordenada pela BIREME e composta de bases de dados bibliográficas produzidas pela Rede BVS, como a Literatura Latino-Americano e do Caribe em Ciências da Saúde (LILACS), além da base de dados MEDLINE e outros tipos de fontes de informação; e CINAHL (Quadro 2).

Quadro 1. Elementos da estratégia PICo, descritores e palavras-chave utilizados. Caxias, MA, Brasil, 2020.

\begin{tabular}{|c|c|c|c|c|c|}
\hline \multicolumn{2}{|c|}{ Elementos } & Mesh & Decs & Cinahl & Palavras-chave \\
\hline P & "Pacientes" & "Patients" & "Patients" & "Patients" & "Patients" \\
& & & "Pacientes" & & "Pacientes" \\
& & & "Pacientes" & & "Pacientes" \\
\hline \multirow{2}{*}{ I } & "Perfil de Saúde" & & "Health Profile" & "Health & "Health Profile" \\
& & & "Perfil de Salud" & Profile" & "Perfil de Salud" \\
& & & "Perfil de Saúde" & & "Perfil de Saúde" \\
\hline Co "Diálise Renal" & "Renal Dialysis" & "Renal Dialysis" & "Renal & "Renal Dialysis" \\
& & & "Diálisis Renal" & Dialysis" & "Diálisis Renal" \\
& & & "Diálise Renal" & & "Diálise Renal" \\
\hline
\end{tabular}

Fonte: A própria pesquisa (2020).

O descritor "Health Profile" não foi encontrado na busca Mesh, portanto foi utilizado como palavra chave. 
Quadro 2. Estratégias de busca utilizadas nas bases de dados BIREME, PUBMED e CINAHL. Caxias, MA, Brasil, 2020.

\begin{tabular}{|c|l|c|c|c|}
\hline Base de dados & \multicolumn{1}{|c|}{ Estratégia de Busca } & Resultados & Filtrados & Selecionados \\
\hline $\begin{array}{c}\text { BIREME } \\
\text { Descritores } \\
\text { Decs) }\end{array}$ & $\begin{array}{l}\text { tw:((tw:(pacientes)) AND (tw:(perfil saúde)) AND } \\
\text { (tw:(dialise renal))) }\end{array}$ & 157 & 56 & 8 \\
\hline PubMed & $\begin{array}{l}\text { (("patients"[MeSH Terms] OR "patients"[All Fields]) } \\
\text { AND (("health"[MeSH Terms] OR "health"[All Fields]) } \\
\text { AND profile[All Fields])) AND ("renal dialysis"[MeSH } \\
\text { Terms] OR ("renal"[All Fields] AND "dialysis"[All }\end{array}$ & 440 & 144 & 5 \\
& Fields]) OR "renal dialysis"[All Fields]) & & \\
\hline $\begin{array}{l}\text { CINAHL } \\
\text { (CINAHL }\end{array}$ & Patients AND Health Profile AND Renal Dialysis & 3 & 1 & \\
\hline
\end{tabular}

Fonte: A própria pesquisa (2020).

Como critérios de inclusão utilizaram-se estudos disponíveis em sua totalidade, publicados nos últimos seis anos, de 2015 até 2020, nos idiomas Português, Espanhol e Inglês. Foram excluídos da busca inicial capítulos de livros, resumos, textos incompletos, teses, dissertações, monografias, relatos técnicos e outras formas de publicação que não artigos científicos completos.

A análise para seleção dos estudos foi realizada em duas fases.

$\mathrm{Na}$ primeira, os estudos foram pré-selecionados segundo os critérios de inclusão e exclusão e de acordo com a estratégia de funcionamento e busca de cada base de dados.

Encontrou-se cento e cinquenta e sete (157) estudos como busca geral na BVS, sendo que limitando a busca para artigos com texto completo realizado com humanos nos últimos cinco anos, obteve-se cinquenta e seis (56) estudos, destes foram analisados títulos e resumos onde apenas oito (8) estudos foram condizentes com a questão desta pesquisa.

Na base PUBMED, como busca total foram encontrados quatrocentos e quarenta (440) estudos, aplicando na pesquisa o filtro que limita por texto completo dos últimos cinco anos com humanos, obteve-se cento e quarenta e quatro (144) estudos, destes foram analisados títulos e resumos e teve como resultado final de cinco (5) estudos.

Na CINAHL foram obtidos três (3) estudos como busca geral, sendo que limitando a busca para artigos com texto completo realizado nos últimos cinco anos com humanos, obteve-se um (1) estudo, sendo que o mesmo não estava condizente com a questão desta pesquisa após a análise dos títulos e resumos.

$\mathrm{Na}$ segunda fase os estudos foram analisados quanto ao potencial de participação no estudo, avaliando o atendimento à questão de pesquisa, bem como, o tipo de investigação, objetivos, amostra, método, resultados e conclusão, resultando, ao final, em treze (13) artigos incluídos na pesquisa.

Quanto a análise e interpretação dos resultados foram analisadas as informações coletadas nos artigos científicos e criadas categorias analíticas que facilitou a ordenação e a sumarização de cada estudo. Essa categorização foi realizada de forma descritiva, indicando os dados mais relevantes para o estudo.

A pesquisa levou em consideração os aspectos éticos da pesquisa quanto às citações dos estudos, respeitando a autoria das ideias, os conceitos e as definições presentes nos artigos incluídos na revisão.

Optou-se pela análise em forma estatística e de forma de texto, utilizando cálculos matemáticos e inferências, que foram apresentados em quadros e tabelas para facilitar a visualização e compreensão. As evidências científicas foram classificadas segundo os níveis propostos por Bork (2005). 


\section{Resultados e Discussão}

Dos treze (13) estudos incluídos nesta revisão, cinco estavam na língua inglesa (61\%), sete na língua portuguesa (53\%) e um na língua Espanhola (7\%). A maioria das publicações foram concentradas no ano de 2018 (30\%) com abordagem quantitativa (100\%); o nível de evidência predominante foi composto principalmente por estudos transversais (84,62\%). O Brasil foi o país com mais estudos incluídos (61\%) (Tabela 1).

Tabela 1. Análise descritiva das produções científicas acerca da caracterização clínica epidemiológica de pacientes que fazem terapia de hemodiálise. Caxias, MA, Brasil, 2020. (n=13).

\begin{tabular}{|c|c|c|}
\hline VARIAVEIS & $\mathbf{N}$ & $\%$ \\
\hline \multicolumn{3}{|l|}{ Base de dados } \\
\hline CINAHL & - & - \\
\hline BIREME & 8 & 61,54 \\
\hline PUBMED & 5 & 38,46 \\
\hline \multicolumn{3}{|l|}{ Abordagem do estudo } \\
\hline Quantitativo & 13 & 100,0 \\
\hline Qualitativo & - & - \\
\hline \multicolumn{3}{|l|}{ Ano } \\
\hline 2015 & 2 & 15,38 \\
\hline 2016 & 2 & 15,38 \\
\hline 2017 & 2 & 15,38 \\
\hline 2018 & 4 & 30,77 \\
\hline 2019 & 3 & 23,08 \\
\hline \multicolumn{3}{|l|}{ Periódicos } \\
\hline Rev. Online de pesquisa cuidado é fundamental & 1 & 7,69 \\
\hline Rev. Baiana de Enfermagem & 1 & 7,69 \\
\hline Cogitare Enferm & 1 & 7,69 \\
\hline Rev enferm UFPE on line & 5 & 38,46 \\
\hline Pan African Medical Journal & 1 & 7,69 \\
\hline Rev. Hindawi & 1 & 7,69 \\
\hline British Medical Journal & 2 & 15,38 \\
\hline Nephrology & 1 & 7,69 \\
\hline \multicolumn{3}{|l|}{ Idioma } \\
\hline Inglês & 5 & 38,46 \\
\hline Espanhol & 1 & 7,69 \\
\hline Português & 7 & 53,96 \\
\hline \multicolumn{3}{|l|}{ Pais } \\
\hline Brasil & 8 & 61,54 \\
\hline Tunísia & 1 & 7,69 \\
\hline Ruanda & 1 & 7,69 \\
\hline Tanzania & 1 & 7,69 \\
\hline Australia & 1 & 7,69 \\
\hline Etiópia & 1 & 7,69 \\
\hline \multicolumn{3}{|l|}{ Delineamento da pesquisa } \\
\hline Estudo de coorte & 2 & 15,38 \\
\hline Estudo transversal & 11 & 84,62 \\
\hline \multicolumn{3}{|l|}{ Classificação da evidencia } \\
\hline Três & 2 & 15,38 \\
\hline Seis & 11 & 84,62 \\
\hline
\end{tabular}

Legenda: \% = percentual; $\mathrm{N}$ = número. Fonte: A própria pesquisa (2020).

O Quadro 3 mostra a distribuição dos estudos segundo autores e anos de publicação em ordem decrescente, o autor; título; tipo de estudo; e objetivos.

Os estudos incluídos nesta revisão destacaram uso de álcool e drogas como comportamento de risco entre os participantes (Santos et al., 2018; Venuthurupalli et al., 2019). No que tange ao tempo de tratamento de hemodiálise, a média 
Research, Society and Development, v. 10, n. 4, e37110414087, 2021

(CC BY 4.0) | ISSN 2525-3409 | DOI: http://dx.doi.org/10.33448/rsd-v10i4.14087

foi de 4,7 anos (Santana et al., 2019; Oliveira, Silva, Ferreira, \& Skalinski, 2015; Mendonça, Dantas, Andrade, Segato, \& Torres, 2015; Silva et al., 2017; Santos et al., 2018; Piccin et al., 2018; Igiraneza, Ndayishimiye, Nkeshimana, Dusabe Jambo, \& Ogbuagu, 2018; Sylvanus et al., 2019; Venuthurupalli et al., 2019).

Quanto ao uso de medicamentos pelos pacientes em tratamento de hemodiálise, os mais consumidos pelos pacientes foram: vitaminas, diuréticos, analgésicos, hipoglicemiantes, ansiolíticos, antiarrítmicos, anti-inflamatórios, antiparasitários e sedativos (Santos et al., 2018; Silva et al., 2017; Guimarães et al., 2016).

Em relação ao tipo de acesso, a fístula arteriovenosa (FAV) foi a mais prevalente nos estudos analisados (Santana et al., 2019; Mendonça et al., 2015; Oliveira et al., 2017; Santos et al., 2018; Piccin et al., 2018; Guimarães et al., 2016). 
Quadro 3. Publicações incluídas segundo o título do artigo, autor, objetivo principal, perfil amostral e principais resultados. Caxias, MA, Brasil, 2020. $(\mathrm{n}=13)$.

\begin{tabular}{|c|c|c|c|c|c|}
\hline $\begin{array}{c}\mathrm{N}^{\circ} \text { de ordem } \\
\text { e base }\end{array}$ & Título do artigo & Autor/ ano & $\begin{array}{c}\text { Delineamento } \\
\text { do estudo/ }\end{array}$ & Objetivo principal & Perfil amostral \\
\hline 01 BIREME & $\begin{array}{l}\text { Perfil dos pacientes submetidos a } \\
\text { tratamento hemodialítico em uma clínica } \\
\text { em Teresina. }\end{array}$ & $\begin{array}{c}\text { Santana et al., } \\
2019\end{array}$ & $\begin{array}{c}\text { Estudo } \\
\text { transversal }\end{array}$ & $\begin{array}{l}\text { Caracterizar a amostra do estudo de acordo com } \\
\text { os dados socioeconômicos; identificar doença de } \\
\text { base e tempo de hemodiálise; identificar o tipo } \\
\text { de acesso no tratamento hemodialítico. }\end{array}$ & $\begin{array}{l}\text { O trabalho permitiu realizar } \\
\text { um levantamento censitário } \\
\text { nos } 271 \text { prontuários existentes } \\
\text { na clínica. }\end{array}$ \\
\hline 02 BIREME & $\begin{array}{l}\text { Perfil dos pacientes renais crônicos em } \\
\text { tratamento hemodialitico. }\end{array}$ & $\begin{array}{l}\text { Oliveira et al. } \\
\text { (2015) }\end{array}$ & $\begin{array}{l}\text { Estudo } \\
\text { transversal }\end{array}$ & $\begin{array}{l}\text { Conhecer o perfil dos pacientes renais crônicos } \\
\text { residentes em Itabuna em tratamento } \\
\text { hemodialítico no ano de } 2012 \text {. }\end{array}$ & $\begin{array}{l}\text { A amostra deste trabalho foi } \\
\text { composta por } 63 \text { pacientes } \\
(25 \%) \text {. }\end{array}$ \\
\hline 03 BIREME & $\begin{array}{l}\text { Perfil Sociodemográfico e clínico de } \\
\text { idosos submetidos à hemodiálise. }\end{array}$ & $\begin{array}{l}\text { Mendonça et } \\
\text { al. } \\
\text { (2015) }\end{array}$ & $\begin{array}{c}\text { Estudo } \\
\text { transversal }\end{array}$ & $\begin{array}{l}\text { Caracterizar os pacientes idosos submetidos à } \\
\text { terapia hemodialítica, quanto aos aspectos } \\
\text { sociodemográficos e clínicos, em dois centros } \\
\text { de nefrologia da cidade de Natal, nordeste do } \\
\text { Brasil, no período de janeiro a março de } 2014 \text {. }\end{array}$ & $\begin{array}{l}\text { A amostra correspondeu a } 81 \\
(48,9 \%) \text { pacientes que } \\
\text { atenderam aos critérios de } \\
\text { seleção. }\end{array}$ \\
\hline 04 BIREME & $\begin{array}{l}\text { Terapia renal substitutiva: perfil } \\
\text { sociodemografico e clínico laboratorial de } \\
\text { pacientes de um serviço de hemodiálise }\end{array}$ & $\begin{array}{l}\text { Silva et al. } \\
\quad(2017)\end{array}$ & $\begin{array}{l}\text { Estudo } \\
\text { transversal }\end{array}$ & $\begin{array}{l}\text { Descrever o perfil sociodemográfico e clínico } \\
\text { laboratorial de pacientes com Doença Renal } \\
\text { Crônica em hemodiálise. }\end{array}$ & $\begin{array}{l}102 \text { pacientes, em tratamento } \\
\text { hemodialítico. }\end{array}$ \\
\hline 05 BIREME & $\begin{array}{l}\text { Perfil socioeconômico e clínico dos } \\
\text { pacientes em programa hemodialitico. }\end{array}$ & $\begin{array}{l}\text { Oliveira et al. } \\
\text { (2017) }\end{array}$ & $\begin{array}{c}\text { Estudo } \\
\text { transversal }\end{array}$ & $\begin{array}{l}\text { Traçar o perfil socioeconômico e clínico de } \\
\text { pacientes em hemodiálise. }\end{array}$ & $\begin{array}{l}74 \text { pacientes, sendo que os } \\
\text { dados coletados foram } \\
\text { referentes ao período } \\
\text { janeiro a junho de } 2012 \text {. }\end{array}$ \\
\hline 06 BIREME & $\begin{array}{l}\text { Perfil do paciente em uso de cateter } \\
\text { venoso central em hemodiálise. }\end{array}$ & $\begin{array}{l}\text { Guimarães et } \\
\text { al. (2016) }\end{array}$ & $\begin{array}{c}\text { Estudo } \\
\text { transversal }\end{array}$ & $\begin{array}{l}\text { Identificar o perfil epidemiológico dos pacientes } \\
\text { submetidos à hemodiálise e os aspectos técnicos } \\
\text { do uso de cateter venoso central temporário de } \\
\text { duplo lúmen (CTDL). }\end{array}$ & $\begin{array}{l}\text { A amostra foi constituída por } \\
57 \text { pacientes. }\end{array}$ \\
\hline 07 BIREME & $\begin{array}{l}\text { Perfil sociodemografico e clínico de } \\
\text { pacientes renais crônicos em hemodiálise. }\end{array}$ & $\begin{array}{l}\text { Piccin et al. } \\
\text { (2018) }\end{array}$ & $\begin{array}{l}\text { Estudo } \\
\text { transversal }\end{array}$ & $\begin{array}{l}\text { Caracterizar os pacientes com doença renal } \\
\text { crônica em hemodiálise quanto aos aspectos } \\
\text { sociodemográficos e clínicos. }\end{array}$ & $\begin{array}{l}\text { A amostra correspondeu a } \\
75,86 \% \text { dos pacientes em } \\
\text { hemodiálise } \\
\text { estipulado. }\end{array}$ \\
\hline 08 BIREME & $\begin{array}{l}\text { Perfil epidemiológico de pacientes renais } \\
\text { crônicos em tratamento hemodiálitico. }\end{array}$ & $\begin{array}{l}\text { Santos et al. } \\
\text { (2018) }\end{array}$ & $\begin{array}{c}\text { Estudo } \\
\text { transversal }\end{array}$ & $\begin{array}{l}\text { Analisar o perfil epidemiológico dos pacientes } \\
\text { com doença renal crônica do serviço de } \\
\text { hemodiálise de uma macrorregião de saúde. }\end{array}$ & $\begin{array}{ll}\text { A amostra } & \text { desta pesquisa } \\
\text { totalizou, } & \text { portanto, } 73 \\
\text { portadores de } & \text { DRC. }\end{array}$ \\
\hline 09 PUBMED & $\begin{array}{l}\text { Profil épidémiologique de l'insuffisance } \\
\text { rénale chronique terminale dans la région } \\
\text { de Sfax. }\end{array}$ & $\begin{array}{l}\text { Chaabouni et } \\
\text { al. (2018) }\end{array}$ & $\begin{array}{l}\text { Estudo } \\
\text { transversal }\end{array}$ & $\begin{array}{l}\text { Descreva o perfil epidemiológico dos casos de } \\
\text { incidentes no governo de Sfax, em um período } \\
\text { de } 10 \text { anos. }\end{array}$ & $\begin{array}{l}1708 \text { casos incidentes em um } \\
\text { período de } 10 \text { anos }\end{array}$ \\
\hline
\end{tabular}




\begin{tabular}{|c|c|c|c|c|c|}
\hline 10 PUBMED & $\begin{array}{l}\text { Clinical profile and outcome of patients } \\
\text { with acute kidney injury requiring dialysis } \\
\text { an experience from a haemodialysis unit in } \\
\text { a developing country. }\end{array}$ & $\begin{array}{l}\text { Ibrahim, } \\
\text { Ahmed, Kedir } \\
\text { e Bekele } \\
(2016)\end{array}$ & $\begin{array}{c}\text { Estudo } \\
\text { transversal }\end{array}$ & $\begin{array}{l}\text { Descrever o perfil clínico e os resultados de } \\
\text { adultos Pacientes com lesão renal aguda (LRA) } \\
\text { tratados com hemodiálise intermitente no centro } \\
\text { de diálise do SPHMMC. }\end{array}$ & $\begin{array}{l}\text { Total de } 243 \text { pacientes foi } \\
\text { submetido a diálise de } 1 \text { de } \\
\text { agosto de } 2013 \text { a } 1 \text { de } \\
\text { fevereiro de } 2015 \text {. }\end{array}$ \\
\hline 11 PUBMED & $\begin{array}{l}\text { Clinical Profile and Outcome of Patients } \\
\text { with Acute Kidney Injury Requiring } \\
\text { Hemodialysis: Two Years' Experience at a } \\
\text { Tertiary Hospital in Rwanda. }\end{array}$ & $\begin{array}{l}\text { Igiraneza et al. } \\
\text { (2018) }\end{array}$ & $\begin{array}{c}\text { Estudo } \\
\text { transversal }\end{array}$ & $\begin{array}{l}\text { Avaliar o impacto da introdução da hemodiálise } \\
\text { (HD) nos resultados de pacientes com LRA em } \\
\text { Ruanda. }\end{array}$ & 82 pacientes. \\
\hline 12 PUBMED & $\begin{array}{l}\text { Profile and outcome of patients with } \\
\text { emergency complications of renal failure } \\
\text { presenting to an urban emergency } \\
\text { department of a tertiary hospital in } \\
\text { Tanzania. }\end{array}$ & $\begin{array}{c}\text { Sylvanus et al. } \\
\text { (2019) }\end{array}$ & $\begin{array}{l}\text { Estudo de } \\
\text { coorte }\end{array}$ & $\begin{array}{l}\text { Descrever o perfil, estratégias de manejo e } \\
\text { resultados de pacientes com insuficiência renal } \\
\text { que apresentavam indicações para diálise } \\
\text { emergente para um departamento de emergência } \\
\text { urbano (DE) em um hospital público terciário na } \\
\text { Tanzânia. }\end{array}$ & 3013 pacientes. \\
\hline 13 PUBMED & $\begin{array}{l}\text { Chronic kidney disease, Queensland: } \\
\text { Profile of patients with chronic kidney } \\
\text { disease from regional Queensland, } \\
\text { Australia: A registry report. }\end{array}$ & $\begin{array}{l}\text { Venuthurupalli } \\
\text { et al. (2019) }\end{array}$ & $\begin{array}{l}\text { Estudo de } \\
\text { coorte }\end{array}$ & $\begin{array}{l}\text { Apresentar o perfil da coorte da DRC recrutada } \\
\text { no Registro CKD.QLD do Toowoomba } \\
\text { Hospital, o primeiro relatório abrangente sobre } \\
\text { uma população em pré-diálise da região da } \\
\text { Austrália. }\end{array}$ & Total de 1051 pacientes. \\
\hline
\end{tabular}

Fonte: A própria pesquisa (2020). 
Os treze estudos analisados fizeram caracterização sociodemográfica e clínica. A idade das pessoas com DRC variou de 20 a 59 anos (Santana et al., 2019; Oliveira et al., 2015; Mendonça et al., 2015; Silva et al., 2017; Oliveira et al., 2017; Santos et al., 2018; Piccin et al., 2018; Guimarães et al., 2016; Chaabouni et al., 2018; Venuthurupalli et al., 2019; Ibrahim et al., 2016; Igiraneza et al., 2018; Sylvanus et al., 2019). Entre os estudos que reportaram o sexo dos pacientes, o sexo masculino foi o mais predominante (Santana et al., 2019; Oliveira et al., 2015; Silva et al., 2017; Oliveira et al., 2017; Santos et al., 2018; Piccin et al., 2018; Guimarães et al., 2016; Chaabouni et al., 2018; Venuthurupalli et al., 2019; Ibrahim et al., 2016; Igiraneza et al., 2018).

Quanto à escolaridade, evidenciou-se baixa escolaridade com níveis menores que o ensino médio (Oliveira et al., 2015; Mendonça et al., 2015; Silva et al., 2017; Oliveira et al., 2017; Piccin et al., 2018; Guimarães et al., 2016). Em relação à renda mensal a maior parte dos participantes nos estudos analisados possuíam em média até 1,3 salários mínimos (Santana et al., 2019; Oliveira et al., 2015; Mendonça et al., 2015; Silva et al., 2017; Oliveira et al., 2017; Santos et al., 2018; Piccin et al., 2018).

No que diz respeito às queixas associadas a hemodiálise, a prevalência foram alterações físicas, cefaleia, câimbras, náuseas, vômitos, e alterações mentais, o que representa alterações nos hábitos de vida, restrições nas atividades cotidianas e diminuição da participação social (Silva et al., 2017; Oliveira et al., 2017; Santos et al., 2018; Piccin et al., 2018; Guimarães et al., 2016; Venuthurupalli et al., 2019; Igiraneza et al., 2018; Ibrahim et al., 2016; Santana et al., 2019).

As principais etiologias associadas ao desenvolvimento de DRC e suas comorbidades, as patologias que prevaleceram foram nefropatia diabética, doença renovascular, incluindo hipertensão arterial e diabetes melittus (Venuthurupalli et al., 2019; Ibrahim et al., 2016; Santana et al., 2019; Oliveira et al., 2015; Mendonça et al., 2015; Silva et al., 2017; Oliveira et al., 2017; Santos et al., 2018; Piccin et al., 2018; Guimarães et al., 2016; Igiraneza et al., 2018).

Este estudo determinou a caracterização clínica epidemiológica dos pacientes renais crônicos, e avaliou as variáveis sociodemográficas e clinicas desses pacientes. A DRC atualmente é vista na saúde pública como um dos principais problemas, sendo causa de diversas mortes. Frequentemente os idosos são os mais acometidos; entretanto, no tempo atual, existem evidências de que o número de pessoas jovens com problemas crônicos de saúde tem aumentado.

\section{Características sociodemográficas dos pacientes em tratamento de hemodiálise}

Os achados relacionados às características sociodemográficas e clinicas dos estudos analisados mostram uma prevalência na faixa etária de 20 a 59 anos. Corroborando com o estudo realizado por Ferreira (2016), sobre o perfil epidemiológico dos pacientes com DRC em Santa Cruz do Sul, em que houve um maior percentual nesta faixa etária de idade. Esses dados apresentam similaridades nos achados de Marçal, Rêgo, Paiano e Radovanovic (2019).

A literatura Internacional na área também revela essa prevalência. O estudo de Gesualdo et al. (2017) realizado em um Serviço de Substituição Renal, evidenciou que a maioria dos pacientes com DRC eram de idade média de 22 a 54 anos.

Os achados relacionados ao sexo predominante, evidenciou que estudos comparativos entre homens e mulheres, têm validado o caso de que os homens estão mais sujeitos às enfermidades, principalmente as doenças graves e crônicas, e que tendem a morrer de forma mais precoce que as mulheres; sendo assim, constatou-se o sexo masculino predominante, corroborando com Ferreira (2016). Em contrapartida, os estudos divergem com o estudo realizado por Conceição, Marinho, Costa, Silva e Lira (2019), apontando prevalência em 50\% de pacientes do sexo feminino submetidos à hemodiálise, apresentando similaridades com o estudo de Batista, Caminha, Silva e Sales (2016).

Com relação ao estado conjugal dos pacientes analisados pelos estudos revisados, houve predomínio de casados, pois ter um companheiro auxilia no enfrentamento das dificuldades da melhor forma possível, sendo assim o estado civil tem forte influência na vida dos pacientes, corroborando com Lemos, Lima, Nascimento e Lira (2015) e Conceição et al. (2019). 
Nos estudos analisados foi constatado que a grande parte dos participantes da pesquisa tinha baixa escolaridade e ensino fundamental incompleto. Corroborando com o estudo semelhante de Jesus et al. (2018) e Porto et al. (2019) nos quais foi retratado que indivíduos com nível de escolaridade mais baixo tendem a sentir mais profundamente o abalo da doença renal crônica em suas atividades cotidianas, ou além disso, podem não seguir ao tratamento ou realiza-lo de forma inadequada.

Em relação à renda, na sua maioria, evidenciou-se que os participantes dos estudos revisados possuem uma renda de até um salário mínimo, procedente dos benefícios ofertados pelo governo por não conseguirem prosseguir com suas atividades por motivo das limitações físicas e também do tempo dedicado ao tratamento. Corroborando, portanto, com dados da literatura de Ferreira (2016) e Ferreira et al. (2018) que indicam que mais de 50\% dos indivíduos eram aposentados ou pensionistas.

No que diz respeito a religião dos pacientes, a maioria da população do estudo era formada por católicos. Segundo Ferreira et al. (2018), em relação à religião, os participantes que praticavam alguma religião apresentavam melhor qualidade de vida, em relação àqueles que não são praticantes de nenhuma crença. Corroborando, portanto, com Conceição et al. (2019), em que é interessante observar que níveis mais elevados de religiosidade indicaram maior felicidade.

\section{Queixas associadas à hemodiálise}

A ocorrência de queixas ou complicação relacionadas a hemodiálise de acordo com os estudos revisados foram alterações físicas, cefaleia, câimbras, náuseas, vômitos e alterações mentais, o que representa alterações nos hábitos de vida, restrições nas atividades cotidianas e diminuição da participação social. Eduardo et al. (2016), apresentaram resultados semelhantes.

De acordo com Santos, Rocha, Ribeiro e Coqueiro (2016), relatam que em relação à alteração física, um dos aspectos salientados pelos entrevistados foi a imagem corporal alterada devido à existência da fístula arteriovenosa (FAV) ou do cateter de duplo-lúmen. Fato esse que leva a modificações na aparência, podendo alterar o estado psicológico do paciente. Os estudos realizados por Manso, Roth e Lopes (2018) afirmam que referente ao tipo e quantidade de trabalho ou atividades cotidianas e o impedimento em realizá-los, colabora para redução da qualidade de vida por causa das alterações musculoesqueléticas frequentes em pacientes submetidos à hemodiálise.

A cefaleia tem causas variadas: como efeito rebote, síndrome do desequilíbrio, por vezes sepse, e cabe ao enfermeiro estar em alerta às que levam o paciente em hemodiálise a manifestar esse sintoma. Esse achado se correlaciona com o estudo de Melo, Ribeiro, Costa e Urel (2015), em que tais pacientes se sentiam fatigados, com dores de cabeça e em outras partes do corpo. De acordo com Eduardo et al. (2016), nas sessões de hemodiálise as câimbras musculares podem estar presentes e geralmente antecedem a hipotensão arterial. Ainda não está bem esclarecida do ponto de vista fisiopatológico, mas é bem associada à diferença de volume entre a ultrafiltração e o reenchimento dos vasos sanguíneos, estando também associada a perda glicose, eletrólitos, etc. Corroborando com Soares et al. (2018), que apresentaram resultados semelhantes.

As náuseas e vômitos são manifestações clínicas de causas distintas que, segundo Santos et al. (2018), acontecem em cerca de $10 \%$ dos tratamentos de hemodiálise. As suas causas fundamentais são: hipotensão, úlcera gástrica, ansiedade, síndrome de desequilíbrio e ingestão de alimentos durante a hemodiálise. Recomenda-se como tratamento o reparo da causa, e se persistir deve ser administrado antiemético.

De acordo com Galvão et al. (2018), a esfera de vida mais afetada é a emocional. A falta de uma assistência psicológica continuada, fora as cautelas dispensadas pela equipe multiprofissional da instituição, aparece como um dado perturbante, no qual se relaciona as questões de saúde mental desses clientes, sendo que, dentre as esferas da vida mais afetadas de acordo com os relato dos pacientes, foi, em maioria, a esfera emocional, tendo como principal sentimento através do o enfrentamento da doença a esperança de cura. 
As limitações nas atividades habituais surgiram fundamentadas nos relatos dos pacientes quanto às mudanças realizadas nas suas rotinas diárias. As doenças degenerativas e crônicas tornam os pacientes dependentes de outras pessoas para o auxílio de suas necessidades. De acordo com Scartolin et al. (2010), após o início do tratamento dialítico, os sujeitos de seu estudo tiveram grandes restrições nas suas atividades de vida diária, transformando não só o cotidiano pessoal, mas de todos os familiares envolvidos. Corroboram com esses achados, os estudos de Santos et al. (2019).

Neste contexto, os pacientes ativos apresentaram melhor percepção de qualidade de vida, em todas dimensões, se comparados aos insuficientemente ativos. A partir do coeficiente de correlação de Spearman, pode-se sugerir que a prática e bons níveis de atividade física tendem a contribuir para melhores escores de qualidade de vida de pacientes em HD (Marques $\&$ Freitas, 2018).

\section{Principais etiologias associadas ao desenvolvimento da DRC e suas comorbidades}

Os achados relacionados apontam em seus artigos que as etiologias e comorbidades encontradas na maioria dos pacientes estudados foram DM e HAS, as quais influenciam no aparecimento da DRC, corroborando com Ferreira (2016), Conceição et al. (2019) e Porto et al. (2019). Nos estudos de Santos (2018), os pacientes apresentaram suscetibilidade aumentada, sendo eles: hipertensos, diabéticos e pacientes com doença cardiovascular, além dos idosos, familiares de pacientes com DRC e pacientes em uso de medicações nefrotóxicas.

A Nefropatia Diabética caracteriza-se pela excreção de quantidades crescentes de albumina na urina, elevação da pressão arterial e falência renal, segundo Pereira et al. (2016), uma das principais complicações relacionadas com a DM. A probabilidade da progressão para nefropatia é maior no DM1. O risco de nefropatia está diretamente relacionado à capacidade do médico controlar simultaneamente e de forma adequada a glicemia, a pressão arterial e taxas lipídicas, bem como, manter a adesão do paciente à terapia medicamentosa prescrita (Guimarães, 2013). Percebe-se que o paciente passa por todo um processo de mudanças após o descobrimento da doença renal, por esse motivo é necessário um período de adaptação para que se alcance qualidade de vida e o mesmo consiga se adequar à nova fase de enfrentamento da doença.

\section{Considerações Finais}

O estudo permitiu traçar a caracterização clínica-epidemiológica dos pacientes em terapia de hemodiálise; e os resultados encontrados evidenciaram a predominância do sexo masculino, casados, renda mensal média de 1,3 salários mínimos. Com relação a escolaridade, a maioria apresentava nível de escolaridade baixa, podendo ser um fator limitante na adesão ao tratamento, como também em segui-lo de forma inadequada. Sobre as queixas associadas a hemodiálise, as mais frequentes foram alterações físicas, cefaleia, câimbras, náuseas, vômitos, alterações mentais e alterações nos hábitos de vida, seguido das etiologias e comorbidades da DRC, houve predomínio da hipertensão arterial e diabetes mellitus.

As limitações deste estudo se devem a ausência de informações relevantes nos estudos analisados, dificultando a obtenção do real conhecimento da caracterização dos usuários do serviço de hemodiálise. A segunda foi o fato de que alguns conteúdos não foram obtidos nas bases de dados eletrônicas, pois os mesmos se apresentavam como pesquisas pagas, e os estudos de interesse encontravam-se incompletos.

Espera-se que essa pesquisa possa contribuir para o alcance de uma verificação contínua da epidemiologia desses pacientes renais crônicos, visando a deteç̧ão precoce da doença, como também as devidas condutas terapêuticas para evitar o desenvolvimento da doença, buscando minimizar o sofrimento desses pacientes, com foco sempre no cuidado integral dos indivíduos e a redução de desfechos desfavoráveis. 
A enfermagem tem papel fundamental na assistência ao paciente com doença renal, não somente nos cuidados diretos, como também nas orientações, pois as ações educativas possibilitam uma melhora no conhecimento dos pacientes, principalmente os de baixa escolaridade, levando sempre em consideração a perspectiva do doente renal, pois é uma importante referência para a gestão dos serviços de saúde, para a construção de protocolos operacionais padrão da equipe multiprofissional, nas recomendações e consensos de especialidades.

\section{Referências}

Albuquerque, J. M. (2017). Marcadores laboratoriais utilizados no diagnóstico da doença renal crônica (Trabalho de Conclusão de Curso). Universidade Federal de Campina Grande, Cuité, PB, Brasil.

Aoyama, E. A., Santos, A. M., Sousa, A. S., Oliveira, D. F., \& Lima, R. N. (2019). Qualidade de vida dos pacientes submetidos ao tratamento de hemodiálise. Revista Brasileira Interdisciplinar de Saúde, 1(3), 73-77.

Batista, A. F., Caminha, M. de F. C., Silva, C. C., \& Sales, C. C. S. (2016). Conhecimento, atitude e prática dos cuidadores de crianças e adolescentes em hemodiálise ou diálise peritoneal. Revista Eletrônica De Enfermagem, 18, e1164.

Bork, A. M. T. (2005). Enfermagem baseada em evidencias. Rio de Janeiro: Guanabara Koonga.

Chaabouni, Y., Yaich, S., Khedhiri, A., Zayen, M. A., Kharrat, M., Kammoun, K.,\& Hachicha, J. (2018). Profil épidémiologique de l'insuffisance rénale chronique terminale dans la région de Sfax. Pan African Medical Journal, 29, 1-9.

Conceição, A. I. C. C., Marinho, C. L. A., Costa, J. R., Silva, R. S., \& Lira, G. G. (2019). Percepções de pacientes renais crônicos na recusa ao transplante renal. Rev. enferm. UFPE on line, 13(3), 664-673, 2019.

Eduardo, M. D., Araújo, G. M. R., Araújo, M. Z., Dantas, M. A. A., \& Alves, M. J. (2016). Atuação da enfermagem nas principais complicações decorrentes do tratamento hemodialítico. Anais do Congresso Brasileiro de Ciências da Saúde, Campina Grande, SP, Brasil, 1.

Ferreira, C., Guanilo, M. E. E., Silva, D. M. G. V., Gonçalves, N., Boell, J. E. W., \& Mayer, B. L. D. (2018). Avaliação de esperança e resiliência em pessoas em tratamento hemodialítico. Revista de Enfermagem da Ufsm, 8(4), 702-716.

Ferreira, M. J. C. (2016). O gerenciamento do cuidado de enfermagem na complexidade da adaptação da pessoa com doença renal crônica em hemodiálise (Dissertação). Universidade Federal do Rio de Janeiro, Escola de Enfermagem Anna Nery, Rio de Janeiro, RJ, Brasil.

Formiga, N.F.B. (2015). Análise de sobrevida e das covariáveis a ela associada de pacientes em diálise do estado de Pernambuco no período de 2008 a 2012. 2015 (Dissertação). Centro de Pesquisas Aggeu Magalhães, Fundação Oswaldo Cruz, Recife, PE, Brasil.

Galvão, A.A.F., Silva, E.G., \& Santos, W.L. (2018). As dificuldades encontradas pelos pacientes com insuficiência renal crônico ao iniciar o tratamento. Revista de Iniciação Científica e Extensão, 2(4), 180-189.

Gesualdo, G. D., Duarte, J. G., Zazzetta, M. S., Kusumota, L., Say, K. G., Pavarini, S. C. I., \& Orlandi, F. S. (2017). Cognitive impairment of patients with chronic renal disease on hemodialysis and its relationship with sociodemographic and clinical characteristics. Dementia \& Neuropsychologia, 11(3), 221-226.

Guimarães, D. (2013). Avaliação de lesões renais por meio do rastreamento de pacientes cadastrados no programa HIPERDIA da região forte de São João, Vitória-ES (Trabalho de Conclusão de Curso). Faculdade Católica Salesiana do Espírito Santo, Vitória, ES, Brasil.

Guimarães, G. L., Goveia, V. R., Mendonza, I. Y. Q., Corrêa, A. R., Matos, S. S., \& Guimarães, J. O. (2016). Perfil do paciente em uso de cateter venoso central em hemodiálise. Rev enferm UFPE on line, 10(12), 4434-42.

Ibrahim, A., Ahmed, M. M., Kedir, S., \& Bekele, D. (2016). Clinical profile and outcome of patients with acute kidney injury requiring dialysis an experience from a haemodialysis unit in a developing country. BMC Nephrology, 17(1), 1-5.

Igiraneza, G., Ndayishimiye, B., Nkeshimana, M., Dusabe Jambo, V., \& Ogbuagu, O. (2018). Clinical Profile and Outcome of Patients with Acute Kidney Injury Requiring Hemodialysis: two years `: experience at a tertiary hospital in rwanda: Two Years’ Experience at a Tertiary Hospital in Rwanda. Biomed Research International, 2018, 1-6.

Jesus, N. M., Souza, G. F., Mendes-Rodrigues, C., Almeida Neto, O. P., Rodrigues, D. D. M., \& Cunha, C. M. (2019). Qualidade de vida de indivíduos com doença renal crônica em tratamento dialítico. Brazilian Journal of Nephrology, 41(3), 364-374.

Lemos, K. C. R., Lima, F. M., Nascimento, K. S., \& Lira, M. N. (2015). Current scenario of Nephrology Nursing and Recife Metropolitan Region. Revista de Pesquisa: Cuidado é Fundamental Online, 7(2), 2349-2361.

Manso, M. E. G., Roth, M. C., \& Lopes, R. G. C. (2018). Convivendo com a doença renal: entre ditos e não ditos. Revista Longeviver, 58 , $108-144$.

Marçal, G. R., Rêgo, A. S., Paiano, M., \& Radovanovic, C.A.T. (2019). Qualidade de vida de pessoas com doença renal crônica em hemodiálise. Rev Fun Care Online, 11(4), 908-913.

Marques, R. V. S., \& Freitas, V. L. (2018). Importância da assistência de enfermagem no cuidado ao paciente transplantado renal. Revista de Enfermagem Ufpe On Line, 12(12), 3436-44. 
Melo, S. P., Ribeiro, R. L. R., Costa, A. L. R. C., \& Urel, D. R. (2015). Community impact of integrative therapy for renal patients people during session hemodialysis. Revista de Pesquisa: Cuidado é Fundamental Online, 7(2), 2200-2214.

Mendonça, A. E. O., Dantas, J. G., Andrade, D. A., Segato, C. T., \& Torres, G. V. (2015). Perfil sociodemográfico e clínico de idosos submetidos à hemodiálise. Cogitare Enfermagem, 20(1), 60-6.

Neves, P. D. M. M., Sesso, R. C. C., Thomé, F. S., Lugon, J. R., \& Nasicmento, M. M. (2020). Brazilian Dialysis Census: analysis of data from the 2009-2018 decade. Brazilian Journal Of Nephrology, 42(2), 191-200.

Oliveira, C. S., Silva, E. C., Ferreira, L. W., \& Skalinski L., M. (2015). Perfil dos pacientes renais crônicos em tratamento hemodialítico. Revista Baiana de Enfermagem, 29(1), 42-49.

Oliveira, D. P. S., Lopes, M. L. H., Silva, G. A. S., Sousa, S. M. A., Dias, R. S., \& Silva, L. V. M. (2017). Socioeconomic and clinical profile of patients in hemodialytic program. Journal of Nursing UFPE on line, 11(11), 4607-4616.

Oliveira, F. B. M., Costa, A. C. A. L., Alves, D. L., França, J. F., Macedo, M. S., \& Santos, R. D. (2016). Relação entre a sobrecarga de trabalho e erros de administração de medicação na assistência hospitalar. Revista Ciências \& Saberes, 2(2), 325-334.

Pereira, A. F. B., Kapper, C. P., Biondo, G., David, M., Russowsky, V. A., \& Agra, H. (2016). Perfil epidemiológico de pacientes portadores de doença renal crônica terminal em programa de hemodiálise em clínica de Santa Cruz do Sul-RS. Proceedings do Congresso Gaúcho de Clínica Médica, Santa Cruz do Sul, $\mathrm{RS}, 13$

Piccin, C., Girardon-Perlini, N. M. O., Coppetti, L. C., Cruz, T. H., Beuter, M., \& Burg, G. (2018). Sociodemographic and clinical profile of chronic kidney patients in hemodialysis. Journal of Nursing UFPE on line, 12(12), 3212-3220.

Porto, A. O., Leal, C. B. M., Barbosa, C. B., Souza, D. A., Boa Sorte, E. T., \& Cruz, S. P. L. (2019). Impacto da hemodiálise nas escórias nitrogenadas séricas. Journal of Nursing UFPE/Revista de Enfermagem UFPE, 13(2), 330-337.

Santana, E. C., Silva, M. S. C., Silva, T. R. G., Oliveira, A. D. S., Ribeiro, I.P., \& Madeira, M. Z. A. (2019). Perfil dos pacientes submetidos a tratamento hemodialítico em uma clínica em Teresina. Rev Fun Care Online, 11(1), 142-146.

Santos, K. K., Lucas, T. C., Glória, J. C. R., Pereira Júnior, A. C., Ribeiro, G. C., \& Lara, M. O. (2018). Epidemiological profile of chronic renal patients in treatment. Journal of Nursing UFPE on line, 12(9), 2293-2300.

Santos, R.P.R. (2018). Autoeficácia e Adesão ao tratamento de indivíduos com doença Renal Crônica em tratamento Hemodialítico (Trabalho de Conclusão de Curso). Universidade Federal de Uberlândia, MG, Uberlândia, Brasil.

Santos, W. N., Rocha, F. C. V., Ribeiro, I. A. P., \& Coqueiro, J. M. (2016). Atuação do enfermeiro nas complicações decorrentes do transplante renal: uma revisão de literatura. Revista Uningá review, 25(1), 131-142.

Scatolin, B. E., Vechi, A. P., Ribeiro, D. F., Bertolin, D. C., Canova, J. C. M., Cesarino, C. B., Ribeiro, R. C. H. M. (2010). Atividade de vida diária dos pacientes em tratamento de diálise peritoneal intermitente com cicladora. Arq Ciênc Saúde [Internet], 17(1), 15-21.

Silva, F., Bettinelli, L. A., Bortoluzzi, E. C., Doring, M., Fortes, V. L. F., \& Dobner, T. (2017). Terapia renal substitutiva: perfil sociodemográfico e clínico laboratorial de pacientes de um serviço de hemodiálise. Rev enferm UFPE on line, 11(9), 3338-45.

Soares, F. C., Aguiar, I. A., Carvalho, N. P. F., Carvalho, R. F., Torres, R. A., Segheto, W., . . . \& Costa, J. A. (2018). Prevalência de hipertensão arterial e diabetes mellitus em portadores de doença renal crônica em tratamento conservador do serviço ubaense de nefrologia. Revista Científica FAGOC-Saúde, 2(2), 21-26.

Sylvanus, E., Sawe, H. R., Muhanuzi, B., Mulesi, E., Mfinanga, J. A., Weber, E. J., \& Kilindimo, S. (2019). Profile and outcome of patients with emergency complications of renal failure presenting to an urban emergency department of a tertiary hospital in Tanzania. Bmc Emergency Medicine, 19(1), 1-8.

Venuthurupalli, S. K., Healy, H., Fassett, R., Cameron, A., Wang, Z., \& Hoy, W. (2019). Chronic kidney disease, Queensland: profile of patients with chronic kidney disease from regional queensland, australia: Profile of patients with chronic kidney disease from regional Queensland, Australia. Nephrology, 24(12), $1257-1264$. 